This is a postprint version of the following article: Portero-Tresserra, Marta; Martí-Nicolovius, Margarita; Gillazo-Blanch, Gemma; Boadas-Vaello, Pere; Vale-Martínez, Anna (2013). DCycloserine in the basolateral amygdala prevents extinction and enhances reconsolidation of odorreward associative learning in rats. Neurobiology of learning and memory. Vol. 100, p. 1-11. ISSN 1074-7427 DOI: 10.1016/j.nlm.2012.11.003

\title{
D-cycloserine in the basolateral amygdala prevents extinction and enhances reconsolidation of odor-reward associative learning in rats
}

\author{
Marta Portero ${ }^{1}$ \\ Margarita Martí-Nicolovius 1 \\ Gemma Guillazo-Blanch ${ }^{1}$ \\ Pere Boadas-Vaello ${ }^{1,2}$ \\ Anna Vale-Martínez 1,3
}

1 Departament de Psicobiologia i Metodologia de les Ciències de la Salut, Institut de Neurociències,

Universitat Autònoma de Barcelona, Barcelona

(Spain).

Present address:

2 Departament de Ciències Mèdiques, Facultat de Medicina,

Universitat de Girona,

Girona (Spain)

${ }^{3}$ Corresponding author:

Anna Vale-Martínez

Departament de Psicobiologia i Metodologia de les Ciències de la Salut, Institut de Neurociències,

Universitat Autònoma de Barcelona, Barcelona

(Spain).

Telephone: 34935813260

Fax: 34935812001

Anna.Vale@ $\underline{\text { uab.cat }}$ 


\section{Abstract}

It is well established that D-cycloserine (DCS), a partial agonist of the NMDA receptor glycine site, enhances learning and memory processes. Although the effects of DCS have been especially elucidated in the extinction and reconsolidation of aversive behavioral paradigms or drug-related behaviors, they have not been clearly determined in appetitive tasks using natural reinforcers. The current study examined the effects of pre-retrieval intra-basolateral amygdala (BLA) infusions of DCS on the extinction and reconsolidation of an appetitive odor discrimination task. Rats were trained to discriminate between three odors, one of which was associated with a palatable food reward and, twenty minutes prior to extinction learning (experiment 1) or reactivation (experiment 2), they received bilateral intra-BLA infusions of DCS or vehicle. In experiment 1, DCS infusion reduced the rate of extinction learning acquisition, weakened extinction retention in a post-extinction test and enhanced reacquisition of the ODT task. In experiment 2, DCS improved subsequent memory expression in the reconsolidation test performed one day after the reactivation session. Such results indicate the involvement of BLA NMDA receptors in odor-food reward associative memory and suggest that DCS may potentiate the persistence or strength of the original memory trace.

Keywords: D-cycloserine, NMDA receptor, olfactory discrimination, extinction, reacquisition, reconsolidation. 


\section{Introduction}

NMDA-receptor transmission is well implicated in learning processes through its role in the modulation of glutamatergic activity. More specifically, NMDA receptors (NMDARs) agonists have been regarded as pharmacological treatments that enhance learning, and as such, have been considered potential cognitive enhancers (see Villarejo-Rodríguez, Vale-Martínez, GuillazoBlanch, \& Martí-Nicolovius, 2010). D-cycloserine (DCS) is a partial agonist at the glycine recognition site of the NMDARs and has been shown to enhance acquisition, consolidation, relearning, extinction and reconsolidation in several associative learning paradigms (Bustos, Giachero, Maldonado \& Molina, 2010; Curlik \& Shors, 2011; Davis, 2002; Ledgerwood, Richardson \& Cranney, 2005; Lee, Milton \& Everitt, 2006; Rodgers, Harvest, Hassall \& Kaddour, 2011; Villarejo-Rodriguez et al., 2010). The majority of studies have focused on the role of DCS in extinction and reconsolidation, as the modulation of such processes may be useful as a treatment strategy for maladaptive memory or anxiety disorders (Davis, Ressler, Rothbaum \& Richardson, 2006; Hofmann, 2007). Although extinction and reconsolidation are both triggered by memory retrieval, reconsolidation is thought to reinforce or update the expression of the original memory while extinction weakens it, possibly through the formation of a new memory trace involving an inhibitory learning process (de la Fuente, Freudenthal \& Romano, 2011; Duvarci, Mamou \& Nader, 2006; Kindt, Soeter \& Vervliet, 2009). An important determinant of subsequent memory expression following reactivation is the length of the reminder experience with brief cues initiating reconsolidation but longer cues resulting in memory extinction (Pedreira \& Maldonado, 2003; Suzuki et al., 2004; Tronson \& Taylor, 2007).

Recent research has reported the role of DCS in the extinction of learned behavior involving appetitive stimuli, such as drug-seeking behavior, suggesting that systemic administration of DCS facilitates the extinction consolidation of self-administration and conditioned place preference (CPP) associated to different drugs (Botreau, Paolone \& Stewart, 2006; Kelley, Anderson \& Itzhak, 2007; Nic Dhonnchadha et al., 2010; Paolone, Botreau \& Stewart, 2009; Thanos, Bermeo, Wang \& Volkow, 2009; Thanos, Bermeo, Wang \& Volkow, 2011) and enhances the persistence of extinction (Groblewski, Lattal \& Cunningham, 2009). In 
contrast to such reports, a recent study showed that pretreatment with systemic DCS prior to extinction training had no effect on the extinction and subsequent reinstatement of morphineinduced CPP (Lu, Wu, Zhang, Ai \& Li, 2011). Food-motivated tasks have been studied to a lesser degree and also indicate inconsistent results. Although systemic injections of DCS enhanced memory consolidation of latent extinction in a food-reward maze (Gabriele \& Packard, 2007), it slowed down the extinction learning of an appetitive instrumental foodrewarded response (Port \& Seybold, 1998). Also, more recent studies have reported the same treatment to have no effect on the extinction learning of an appetitive operant response reinforced with food (Vurbic, Gold \& Bouton, 2011) or on extinction and spontaneous recovery of conditioned taste aversion when a chronic treatment was involved (Mickley et al., 2012). Such discrepancies may depend on the timing of DCS administration, as the studies using pretraining injections were predominant in showing impairments or no effects on extinction (Lu et al., 2011; Port \& Seybold, 1998; Vurbic et al., 2011). Similarly, there are several reports from human studies suggesting that administration of DCS prior to extinction has no effect on fear conditioning (Klumpers et al., 2012) or alcohol dependence (Watson et al., 2011) and that it enhances craving in the case of cocaine-dependence (Price et al., 2012).

The basolateral amygdala (BLA) is a brain region that has received a great deal of attention in terms of memory extinction and reconsolidation, and especially so in aversive paradigms (Lee et al., 2006; Nader, Schafe \& Le Doux, 2000; Rehberg, Bergado-Acosta, Koch \& Stork, 2010). Previous literature has mainly examined the effects of DCS on contextual or auditory conditioned fear, indicating that systemic and intra-BLA administrations of DCS may potentiate both extinction and reconsolidation (Ledgerwood, Richardson \& Cranney, 2003; Ledgerwood et al., 2005; Lee et al., 2006; Toth et al., 2012; Walker, Ressler, Lu \& Davis, 2002; Yamada, Zushida, Wada \& Sekiguchi, 2009; Yamamoto et al., 2008). Also, DCS in the BLA facilitated the extinction of contextual fear with no effect on the extinction of conditioned taste aversion (Akirav, Segev, Motanis, \& Maroun, 2009). However, there has been very little systematic investigation as to the intra-BLA effects of DCS in the extinction and reconsolidation of appetitive paradigms, particularly those using natural incentives. Although there are no 
reports on the effects of pre-training DCS infusions into the BLA on extinction, there are some studies using post-training infusions that show divergent results. While Torregrossa, Sanchez, \& Taylor, (2010) found no effect in the extinction of cocaine-associated cues, Botreau et al. (2006) reported the facilitation of cocaine-induced CPP extinction formation. As for reconsolidation studies, intra-BLA infusions prior to reactivation facilitated memory reconsolidation of stimulus-cocaine association in a self-administration paradigm (Lee, Gardner, Butler \& Everitt, 2009).

To determine whether extinction and reconsolidation of an appetitive model is modulated by pre-retrieval intra-BLA DCS, we based our experiments on a simple and noninvasive food reward-based olfactory paradigm. This odor discrimination task (ODT) involves a rapidly acquired association between odor and palatable food reward and allows for consistent memory. It entails neither fear nor acute stress and is sensitive to NMDARs manipulation. (Tronel \& Sara, 2003). Thus, infusions of NMDARs antagonists in the prelimbic cortex have been shown to prevent initial ODT consolidation (Tronel \& Sara, 2003) and disrupt reconsolidation when infused into the cerebral ventricles (Torras-Garcia, Lelong, Tronel \& Sara, 2005). However, additional results suggest that pretraining DCS infusions in the prelimbic cortex enhance ODT relearning without affecting initial acquisition or consolidation (VillarejoRodriguez et al., 2010). In addition to the prefrontal cortex, the BLA has also been related to ODT as it is markedly activated following the acquisition of the odor-reward association(Tronel \& Sara, 2002). Nevertheless, its specific role in ODT extinction or reconsolidation processes has yet to be determined.

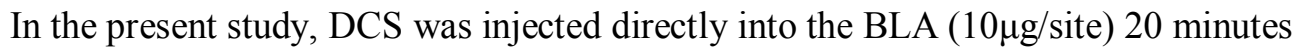
prior to extinction learning (experiment 1) or memory reactivation (experiment 2) of the odorreward association. In experiment 1, a reacquisition session was carried out after extinction training to obtain an indirect measure of the animals' extinction level. While the first experiment involved re-exposing rats to the conditioned stimulus (CS, rewarded odor) in the absence of the unconditioned stimulus (US, chocolate cereal) during 5 consecutive trials, in the 
second experiment, the rats were presented with a brief 90 -sec reminder (rewarded odor), also in the absence of US. Dose and time point were based on previous results suggesting extinction, reconsolidation and relearning facilitation (see 2.3).

\section{Material and Methods}

\subsection{Subjects}

Seventy-two male Wistar rats belonging to our laboratory's breeding stock were used: 22 rats in experiment 1 (mean age $=91 \mathrm{~d}, \mathrm{SD}=5.02$; mean weight $=393.1 \mathrm{~g}, \mathrm{SD}=27.4$ at the beginning of the experiment) and 50 in experiment 2 (mean age $=92.8 \mathrm{~d}, \mathrm{SD}=8.9$; mean weight $=380.20 \mathrm{~g}$, $\mathrm{SD}=41.0$ ). All the rats were single-housed in $50 \times 22 \times 14 \mathrm{~cm}$ plastic-bottomed cages with sawdust bedding, in a room controlled for temperature $\left(20-22^{\circ} \mathrm{C}\right)$ and humidity $(40 \%-70 \%)$. The rats were maintained on a $12 \mathrm{~h}$ light-dark cycle (lights on at 8:00 a.m.), with experiments performed during the light phase of the cycle. Rat-chow pellets (Scientific Animal Food \& Engineering, Augy, France) and water were provided ad libitum with the exception of habituation, acquisition, extinction, reacquisition and reconsolidation test sessions, in which the rats were submitted to a food restriction schedule ( $12 \mathrm{~g} /$ day) to maintain their body weight at $85 \%$ of their free-feeding weight. The animals were handled on a daily basis for 5 minutes and restrained for 2 minutes to accustom them to the injection procedure. All procedures were carried out in compliance with the European Community Council Directive for care and use of laboratory animals (86/609/ECC) and with the Generalitat de Catalunya's authorization (DOGC 2450 7/8/1997, DARP protocol number 5959).

\subsection{Surgery}

Animals were anesthetized with isoflurane and underwent stereotaxic implantation of bilateral chronic guide cannulae in the BLA following procedures explained elsewhere (CarballoMarquez, Vale-Martinez, Guillazo-Blanch \& Marti-Nicolovius, 2009). Each guide cannula comprised one 26-gauge metal tube projecting $7.5 \mathrm{~mm}$ from the pedestal (Plastics One®, Bilaney Consultants GMBH, Germany). The stereotaxic coordinates used for implantation into 
the BLA were: AP: $-2.6 \mathrm{~mm}$ from bregma; ML: $\pm 4.9 \mathrm{~mm}$ from midline, and DV: $-7.5 \mathrm{~mm}$ from cranium surface (Paxinos \& Watson, 1997). Sterile dummy stylets (Plastics One®) were placed into the cannulae to prevent occlusion. After surgery, the rats were replaced in their home cages for seven days prior to behavioral training ( 3 days for recovery, 3 days for food restriction, and 1 day for habituation). During the 7-day recovery period, the rats were handled and weighed on a daily basis and the dummy stylets were changed every other day.

\subsection{Microinfusion Procedure}

Twenty minutes prior to the ODT extinction learning (experiment 1) or reactivation (experiment 2) sessions, the rats were gently restrained while the dummy stylets were removed and replaced with a 33-gauge stainless-steel injector (Plastics One ${ }^{\circledR}$ ) extending $1 \mathrm{~mm}$ below the cannula tips. The injection-to-extinction learning/reactivation interval was the same as in previous research showing a positive effect of DCS on ODT performance (Villarejo-Rodriguez et al., 2010) and similar to that used in other experiments (15 to $30 \mathrm{~min}$ ) reporting facilitation of intra-BLA DCS on extinction (Lee et al., 2006; Mao, Hsiao, \& Gean, 2006; Walker et al., 2002) and reconsolidation (Lee et al., 2009; Yamada et al., 2009). The injectors were connected by polyethylene tubing (Plastics One ${ }^{\circledR}$ ) to two 10- $\mu$ l syringes (SGE Analytical Science, Cromlab S.L. Barcelona, Spain) mounted in an infusion pump (11 Plus Syringe Pump, Harvard Apparatus Inc., USA). DCS (Sigma-Aldrich, Madrid, Spain) was dissolved in PBS (phosphatebuffered saline $0.1 \mathrm{M} \mathrm{pH} \mathrm{7.4)} \mathrm{and} \mathrm{a} \mathrm{dose} \mathrm{of} 10 \mu \mathrm{g}$ / hemisphere was infused in the DCS group rats. The solution was infused bilaterally in a volume of $0.5 \mu 1 /$ hemisphere for 2 minutes. The dose was also determined both on the basis of our previous research with ODT (VillarejoRodriguez et al., 2010) and previous literature relating to intra BLA infusions (Ledgerwood et al., 2003; Mao et al., 2006). The inner cannulae were left in place for 1 minute after the infusion was complete to allow for diffusion. The rats in the VEH groups received PBS injections under the same conditions.

\subsection{Apparatus}


The training apparatus and behavioral procedures are explained elsewhere (Carballo-Marquez et al., 2007). The training apparatus was a $60 \times 60 \times 40 \mathrm{~cm}$ square box containing three sponges $(8.5 \times 6.5 \times 5.5 \mathrm{~cm})$ with a $3-\mathrm{cm}$ diameter hole cut into the center to a depth of $2.5 \mathrm{~cm}$, placed in glass slide-holders of the same size. The food reinforcement used was a crispy chocolate rice breakfast cereal (Kellogg's, Spain) that was placed at the bottom of the opening in the sponge. Each sponge was infused with an odor that was injected into each of its corners. The odors, vanilla $(0.3 \mathrm{ml})$, orange $(0.6 \mathrm{ml})$ and anise $(0.2 \mathrm{ml})$ (Vahiné, Ducros S.A., Sabadell, Spain), were previously tested in a pilot study in which the rats showed no particular preference. All behavioral sessions were recorded by a video camera (JVC, Everio Model GZ-X900) connected to a monitor.

\subsection{Behavioral Procedures in Experiment 1: ODT Extinction}

\subsubsection{Habituation sessions}

The rats were food-deprived for five days prior to three habituation sessions in which they were given free access to the reinforcement in a plastic bottomed cage $(50 \times 22 \times 14 \mathrm{~cm})$. After consuming ten pieces of cereal, they were placed in the training box, without the reinforcement, and allowed to explore it for 15 minutes. Once the rats had recovered from surgery, they were once again food-deprived and submitted to an identical habituation session. On the same day, the rats were also adapted to a mock infusion protocol (no solutions injected) in order to minimize any stress associated with the procedure.

\subsubsection{Acquisition session}

One day after the post-surgery habituation, ODT acquisition was carried out in a 4-trial session (see Fig. 2A), in accordance with previously described procedures (Quiroz-Padilla, GuillazoBlanch, Vale-Martinez, Torras-Garcia \& Marti-Nicolovius, 2007). The reinforcement (US, chocolate rice cereal placed at the bottom of the opening in the target sponge) was associated with the same odor across trials $\left(\mathrm{CS}^{+}\right)$, and the rewarded odor was randomly assigned to each rat in a counterbalanced manner. The sponges with the non-rewarded odors did not contain any 
food. Sponges were placed in any three of the four corners of the box, and the position of each odor within the box was changed for each trial according to a previously determined protocol. The rats were placed in the training box, facing the corner without a sponge. After the rats had found and eaten the cereal, they remained in the box for a few seconds before being removed and placed in the intertrial cage for one minute before the following trial. There was a 3-minute maximum period for the rat to find and consume the reinforcement. Failure to find and eat the cereal within this period resulted in the rat's being removed from the training box and placed in the intertrial cage for one minute before the following trial. The rats with latencies longer than three min in each acquisition trial were excluded from the analyses on the grounds that they had not correctly learned the task $(n=2)$. Latency before a correct response (nose-poking into the rewarded sponge) and number of errors were scored as dependent variables. Two different errors were combined: errors of commission (nose-poking into a non-rewarded sponge) and omissions (sniffing the rewarded sponge with no subsequent nose-poking) (Tronel \& Sara, 2003).

\subsubsection{Extinction learning}

Twenty-four hours after the acquisition session, the rats received a bilateral intracerebral infusion of DCS (DCS group) or PBS (VEH group) in the BLA 20 min prior to ODT extinction learning. Extinction learning was carried out in a single 5-trial session (a pilot study showed a good extinction performance after 5 trials), using the same procedure as in the preceding

session, but no food reward was placed in any of the sponges for any of the trials $\left(5\right.$ trials, $\mathrm{CS}^{+}$/ no US). The trials were considered to be complete when the subjects had made the conditioned

response, nose-poking into the previously rewarded sponge $\left(\mathrm{CS}^{+}\right)$, or after three min. The measures considered were the same as in the previous session, but a large number of errors and long latencies to nose-poke into the formerly rewarded sponge were an index of good extinction learning. An extinction criterion was also established: latency, during 2 consecutive trials, to make the formerly correct response $\geq 44 \mathrm{sec}$, which was the mean latency observed in the whole 
first-trial sample (acquisition session) when the animals had not yet learned the task.

\subsubsection{Extinction retention}

Twenty-four hours after the extinction learning session, a free-drug test (extinction retention) similar to the previous session was carried out. The rats were once again placed in the training box and underwent five further extinction trials with no reward in any of the sponges ( 5 trials, $\mathrm{CS}^{+} /$no US).

\subsubsection{Reacquisition session}

Twenty-four hours after extinction retention, a final session, in which the rats were returned to the original learning conditions, was performed. In this session, the rewarded odor was again associated with the cereal, as in the acquisition session (4 trials, $\mathrm{CS}^{+} / \mathrm{US}$ ).

\subsection{Behavioral Procedures in Experiment 2: ODT Reconsolidation}

Behavioral procedures of habituation and acquisition were similar to those described for experiment 1 .

\subsubsection{Reactivation session}

Twenty-four hours after acquisition, the rats received a reactivation session (see Fig. 4A) consisting of a brief exposure $(90 \mathrm{sec})$ to a small sponge (different from the one used in the ODT training) infused with the reinforced odor, within the inter-trial cage, in the same room the acquisition had taken place. No food reward was present during this session. During the session, motor activity and contacts with the sponge were directly observed by the experimenter. Four experimental groups were studied, but only two received the memory reactivation (REACT groups) while the other two remained in a quiet room adjacent to the experimental room (nonREACT group). Twenty minutes prior to the reactivation protocol, the rats from both groups received a bilateral intraBLA infusion of DCS (DCS REACT and DCS nonREACT) or PBS (VEH REACT and VEH nonREACT). 


\subsubsection{Test session}

Twenty-four hours after the reactivation session, the rats were tested in a 4-trial retention/ reconsolidation session using the same procedure as in acquisition (see also Torras-Garcia et al., 2005).

\subsection{Olfactory perception test}

To rule out olfactory impairments due to the infusion of DCS, an additional olfactory perception test was conducted at the end of each experiment (Carballo-Marquez et al., 2007; QuirozPadilla, Guillazo-Blanch, Vale-Martinez \& Marti-Nicolovius, 2006; Wrenn, Harris, Saavedra \& Crawley, 2003). Twenty-four hours prior to the olfactory test, the rats were habituated to butterflavored cookies (Brambly Hedge, Denmark). They were then food-restricted for 24 hours prior to the infusion and the test. Twenty minutes (experiment 1) or 24 hours (experiment 2 ) before the test, they were infused with DCS $(10 \mu \mathrm{g})$ or PBS. The test was conducted in clean rat cages $(50 \times 22 \times 14 \mathrm{~cm})$ and a piece of cookie was buried in one corner of the cage. The rats were then placed in the cage, and the latency to find the buried cookie and commence eating was timed.

\subsection{Histology}

Upon completion of the behavioral study, the rats were deeply anesthetized with an overdose of sodium pentobarbital (Dolethal, $200 \mathrm{mg} / \mathrm{kg}$; Vetoquinol S.A., Madrid, Spain) and perfused transcardially with PBS (pH: 7.4) followed by $4 \%$ paraformaldheyde in $0.1 \mathrm{M}$ PBS at a flow rate of $40 \mathrm{ml} / \mathrm{min}$. Subsequently, the cannulae were carefully removed and brains were postfixed in paraformaldehyde for two hours and then submerged in a $20 \%$ sucrose solution prior to sectioning. Coronal 40- $\mu \mathrm{m}$ sections were cut on a cryostat (Shandom Cryotome FSE, Thermo Electron Corporation, Massachusetts, USA), mounted and processed for acetylcholinesterase histochemistry, essentially as described elsewhere (Paxinos \& Watson, 1997). The sections were examined to verify cannula placement by two independent observers under a light microscope (Olympus BX 41; Olympus Optical CO, LTD, Japan). Microphotographs of the cannula placements were obtained using a digital camera (Olympus DP70). 


\subsection{Data analysis}

In experiment 1 , data were submitted to a mixed analysis of variance using repeated measures (ANOVA; PASW v19) in which the between-factor was group (DCS and VEH) and the withinfactor was session. The session factor consisted of 4 measures: acquisition (the average scores for the 4 trials), extinction learning (the average scores for the 5 trials), extinction retention (the average scores for the 5 trials) and reacquisition (the average scores for the 4 trials). The dependent variables were latencies and number of errors. Any deviations from sphericity were corrected using the Greenhouse-Geisser correction if $p<0.05$, and corresponding contrasts were performed when necessary. Trial-by trial analyses of the learning evolution (latencies and errors) during the extinction learning, extinction retention and reacquisition sessions were performed by means of ANOVA. An additional survival analysis (procedure Kaplan-Meier and Breslow contrasts) was also carried out to analyse and compare the mean number of extinction trials, during learning and retention sessions, required by each experimental group in order to reach the pre-established extinction criterion (see 2.5.3).

In experiment 2, analyses of variance were carried out, with session as a within-subject factor that consisted of 2 measures: acquisition (the average scores for the 4 trials) and test (the average scores for the 4 trials), and drug (DCS and VEH) and reactivation (reactivated vs nonreactivated) as the between-subject factors following a 2x2 design. The dependent variables measured were latencies and errors. Additionally, a trial-by trial analysis of the learning evolution (latencies and errors) during the test session was performed by means of ANOVA with the group factor made up of 4 categories (DCS-REACT, DCS-nonREACT, VEH-REACT, VEH-nonREACT). When between-group differences were analyzed, the Bonferroni correction was used to counteract the problem of multiple comparisons.

Regarding the olfactory perception test, two additional ANOVA analyses (injection-test delay 20 minutes and 24 hours) were applied considering group (DCS and VEH) as the independent variable and latency in finding the buried cookie as the dependent variable. 


\section{Results}

\subsection{Histology (Experiments 1 and 2)}

Only rats with patent microinjector tips within the boundaries of the BLA were included in the analyses. Subjects were only included if their injector tips were located bilaterally within the BLA (in the area delimited by the basomedial amygdala, the lateral amygdala, the basolateral posterior amygdala and the bed nucleus of the stria terminalis) and no tissue damage, caused by the rate or volume of infusions, was detected (Fig. 1A). Specifically, the cannulae were located along different brain coordinates from $2.30 \mathrm{~mm}$ to $3.14 \mathrm{~mm}$ posterior to bregma (Paxinos and Watson 1997) (Fig. 1B-C). Subjects with incorrectly implanted cannulae (located in other amygdalar nuclei) were excluded from the analyses (rats in experiment 1: DCS, $n=2$; rats in experiment 2: DCS-REACT, $\mathrm{n}=2$; DCS-nonREACT, $\mathrm{n}=1$; VEH-REACT, $\mathrm{n}=1$ ).

\subsection{Behavior}

\subsubsection{Experiment 1: ODT Extinction}

The DCS group ( $\mathrm{n}=9$ ) showed significant disruption of ODT extinction learning and memory when compared to the VEH group $(n=9)$, demonstrated by marked differences in latencies and errors in both extinction sessions, and facilitation of ODT reacquisition (shorter latencies and less number of errors), suggesting persistence to nose-poking into the sponge previously reinforced in the acquisition session. The analysis of the latencies (Fig. 2B) showed statistically significant differences between both groups $\left(\mathrm{F}_{(1,16)}=11.424, \mathrm{p}=0.004\right)$. There was also a significant effect of session $\left(\mathrm{F}_{(3,48)}=18.764, \mathrm{p}<0.001\right)$ and group $\times$ session interaction $\left(\mathrm{F}_{(3,48)}\right.$ $=6.512, \mathrm{p}=0.004)$, indicating that the progress of the behavior across the four sessions was different between both groups. Specifically, DCS rats showed shorter latencies to nose-poke the sponge originally associated with reward in extinction learning $(\mathrm{p}=0.001)$, extinction retention test $(\mathrm{p}=0.003$ ) and reacquisition ( $\mathrm{p}=0.042)$. The within-group analysis showed differences in the VEH group between acquisition and extinction learning ( $\mathrm{p}<0.001)$, extinction retention 
$(\mathrm{p}<0.001)$ and reacquisition $(\mathrm{p}=0.024)$, but not in DCS group, indicating that only VEH rats exhibited clear signs of extinction.

The trial-by-trial analysis of the latencies confirmed the previous analyses as it showed a divergent evolution of DCS and VEH groups (Fig. 2C), indicating that both groups started from a similar level (extinction learning session, trial 1: $\mathrm{F}_{(1,16)}=3.116, \mathrm{p}=0.097$ ) and progressively differed through extinction learning (trial $2: \mathrm{F}_{(1,16)}=7.022, \mathrm{p}=0.017$; trial 4: $\left.\mathrm{F}_{(1,16)}=11.572, \mathrm{p}=0.004\right)$, extinction retention (trial 1: $\mathrm{F}_{(1,16)}=4.723, \mathrm{p}=0.045$; trial 3: $\mathrm{F}_{(1,16)}=5.355, \mathrm{p}=0.034 ;$ trial $4: \mathrm{F}_{(1,16)}=15.056, \mathrm{p}=0.001$ and trial 5: $\mathrm{F}_{(1,16)}=9.137$, $\mathrm{p}=0.008)$ and reacquisition (trial 1: $\left.\mathrm{F}_{(1,16)}=6.051, \mathrm{p}=0.026\right)$.

Such results were completed by a survival analysis of the latencies within both extinction sessions (Fig. 3), which indicated that the DCS rats were slower to extinguish their behavior as they needed a higher number of trials to reach the extinction criterion (see 2.5.3.), and also the percentage of DCS subjects achieving the criterion was significantly lower than VEH subjects in extinction learning $\left(\mathrm{X}^{2}=8.837, \mathrm{df}=1, \mathrm{p}=0.003\right)$ and in extinction retention $\left(\mathrm{X}^{2}=4.537, \mathrm{df}=1, \mathrm{p}=0.033\right)$. Thus, after 3 extinction learning trials, $55.5 \%$ of the VEH rats overcame the extinction criterion but none of their DCS counterparts and, by the end of the session, $90 \%$ in the VEH group vs $55.5 \%$ in the DCS group. By the final retention extinction trial, $100 \%$ of the VEH rats had acquired the criterion but only $66.6 \%$ of the rats injected with DCS prior to extinction learning.

The analysis of the number of errors (Fig. 2D) displayed a similar pattern of results to the latency analyses with significant between-group differences $\left(F_{(1,16)}=14.613, p=0.001\right)$. The session factor was not statistically significant $\left(\mathrm{F}_{(3,48)}=0.499, \mathrm{p}=0.685\right)$ but the session $\mathrm{x}$ group interaction showed a tendency toward significance $\left(\mathrm{F}_{(3,48)}=2.028, \mathrm{p}=0.122\right)$. The DCS 
group committed fewer errors than the VEH group in extinction learning $\left(\mathrm{F}_{(1,16)}=9.132\right.$, $\mathrm{p}=0.008)$, extinction retention $\left(\mathrm{F}_{(1,16)}=5.689, \mathrm{p}=0.030\right)$, and reacquisition $\left(\mathrm{F}_{(1,16)}=9.692\right.$, $\mathrm{p}=0.007$ ), indicating a possible resistance to extinction. The within-group analysis indicated that the VEH group increased the number of errors from the acquisition session to the extinction learning ( $\mathrm{p}=0.05)$ while the DCS group did not. There were statistically significant differences in the DCS group ( $\mathrm{p}=0.05$ ) between acquisition and reacquisition but not so in the VEH group. The trial-by-trial analysis of the total number of errors (Fig. 2E) showed statistically significant differences between groups in the third extinction retention trial $\left(\mathrm{F}_{(1,16)}=6.050, \mathrm{p}=0.026\right)$.

Poorer performance in ODT extinction did not seem to be related to changes in olfactory sensitivity since no statistically significant between-group differences were observed when the latency to find a buried sweet-smelling cookie (20 minutes after injection) was analyzed a day after the completion of experimental manipulations (DCS: mean $=34.70$, $\mathrm{SE}=4.78$; VEH: mean=33.40, $\left.\mathrm{SE}=2.90 ; \mathrm{F}_{(1,18)}=0.054, \mathrm{p}=0.819\right)$.

\subsubsection{Experiment 2: ODT Reconsolidation}

The four treatment groups based on the two factors analyzed (drug and memory reactivation) were DCS-REACT ( $\mathrm{n}=12)$, VEH-REACT $(\mathrm{n}=12)$, DCS-nonREACT $(\mathrm{n}=12)$, VEH-nonREACT $(n=10)$. As depicted in Fig. 4B-D, the DCS group exposed to a memory reactivation protocol (DCS-REACT) performed shorter latencies and fewer errors in the test session, suggesting an enhanced ODT reconsolidation. The analyses revealed that the session factor was statistically significant for both latencies $\left(\mathrm{F}_{(1,42)}=21.383, \mathrm{p}<0.001\right)$ and number of errors $\left(\mathrm{F}_{(1,42)}\right.$ $=9.510, \mathrm{p}<0.01)$. As expected, the differences between groups were found in the test; therefore, an additional analysis was carried out for the retention session. The main effects in this session showed that the drug factor was statistically significant for latencies and number of errors $\left(\mathrm{F}_{(1}\right.$, $42)=6.604, \mathrm{p}=0.014$ and $\mathrm{F}_{(1,42)}=10.229, \mathrm{p}=0.003$, respectively), and the reactivation factor 
showed a tendency toward significance for the latencies $\left(\mathrm{F}_{(1,42)}=2.698, \mathrm{p}=0.108\right)$ and number of errors $\left(\mathrm{F}_{(1,42)}=3.788, \mathrm{p}=0.06\right)$. The interaction between drug and reactivation was statistically significant for number of errors $\left(\mathrm{F}_{(1,42)}=4.546, \mathrm{p}=0.039\right)$ but not for latencies $\left(F_{(1,42)}=1.150, p=0.290\right)$. Specifically, statistically significant differences were found between DCS-REACT and VEH-REACT in latencies $(\mathrm{p}=0.012)$ and number of errors $(\mathrm{p}<0.001)$, between DCS-REACT and DCS-nonREACT in latencies $(\mathrm{p}=0.05)$ and number of errors $(\mathrm{p}=0.005)$, and between DCS-REACT and VEH-nonREACT in latencies $(\mathrm{p}=0.006)$ and number of errors $(\mathrm{p}=0.001)$. The trial-by-trial analysis of the latencies in the test session showed a different evolution of the DCS-REACT group with respect to the other three groups (Fig. 4C). There were statistically significant effects of the between-group factor $\left(F_{(3,42)}=3.508\right.$, $\mathrm{p}=0.023)$, and the within factor trial $\left(\mathrm{F}_{(3,126)}=7.180, \mathrm{p}<0.001\right)$ and the interaction showed a tendency toward statistical significance $\left(\mathrm{F}_{(9,126)}=1.750, \mathrm{p}=0.084\right)$. The analyses of the errors (Fig. 4E) showed a similar pattern with a statistically significant between-subject effect $\left(\mathrm{F}_{(3,42)}=6.338, \mathrm{p}=0.001\right)$ and within-subject effect $\left(\mathrm{F}_{(3,126)}=4.965, \mathrm{p}=0.003\right)$ and a tendency toward significance of the interaction factor $\left(\mathrm{F}_{(9,126)}=1.718, \mathrm{p}=0.09\right)$. Specifically, DCSREACT showed shorter latencies in the test session when compared to the other groups (Trial 1: vs DCS-nonREACT, $\mathrm{p}=0.012$; and vs VEH-nonREACT, $\mathrm{p}=0.001$; Trial 2: vs VEH-REACT, $\mathrm{p}=0.05$; Trial 3: vs VEH-REACT, $\mathrm{p}=0.008$; and vs VEH-nonREACT, $\mathrm{p}=0.045$; Trial 4: vs VEHREACT p=0.047; and vs VEH-nonREACT, p=0.043). Also, DCS-REACT showed fewer number of errors (Trial 1: vs DCS-nonREACT $\mathrm{p}=0.04$; Trial 2: vs VEH-REACT, $\mathrm{p}=0.009$; and and vsVEH-nonREACT, $\mathrm{p}=0.017$; Trial 3: vsVEH-REACT, $\mathrm{p}=0.003$; and vs VEH-nonREACT, $\mathrm{p}=0.003)$.

Finally, no statistically significant between-group differences were observed when the latency to find a buried cookie ( 24 hours after injection) was analyzed two days after completing behavioral testing (DCS: mean=45.48, $\mathrm{SE}=2.240$; $\mathrm{VEH}$ : mean=43.96,SE=2.534; 
$\left.F_{(1,42)}=0.202, p=0.655\right)$.

\section{Discussion}

The results of our study indicate that the administration of DCS directly into the BLA hindered extinction and potentiated reconsolidation of odor-food reward associative memory. In the first experiment, the group receiving pre-extinction DCS showed an impaired performance, both in latencies and errors, in extinction learning and 24-h retention. Thus, DCS and VEH rats started extinction learning from a similar level of performance but they diverged across subsequent trials. Furthermore, the number of trials needed to reach the extinction criterion was higher in the DCS group than in the VEH group, and in the drug-free extinction test (24h-retention) $66.5 \%$ of DCS rats acquired the criterion in the last trial vs $100 \%$ of VEH rats. Such resistance to extinction observed in the DCS group was also revealed by its rapid reacquisition of the original learning after extinction training. Also, the DCS rats displayed shorter latencies and fewer errors to make the correct response in the reacquisition than in the initial acquisition, in contrast to VEH rats. As there was no substantial effect of DCS on odor sensitivity, the results would seem to indicate that, in control rats, several exposures to the reinforced odor in the absence of the reward triggered the formation of a new extinction trace encoding the stimuli dissociation. Such new learning was attenuated in rats pretreated with a single injection of DCS into the BLA, as indicated by poorer extinction and enhanced reacquisition, which may be interpreted as a potentiated persistence of the original memory trace.

The findings in experiment 1 agree with other studies in animals failing to show facilitation in the extinction of appetitive tasks, specifically those reinforced with food, when systemic pre-extinction DCS injections were administered (Port \& Seybold, 1998; Vurbic et al., 2011). Similarly, one study demonstrated no positive effect of systemic pre-extinction DCS on ethanol-CPP extinction acquisition, although it did report an enhanced persistence of extinction during reconditioning (Groblewski et al., 2009). Moreover, recent studies in humans have also revealed that DCS administered before extinction had no effects on the extinction of drug dependence (Price et al., 2012; Watson et al., 2011) and on a variety of behavioral disorders 
(Guastella, Lovibond, Dadds, Mitchell, \& Richardson, 2007; Storch et al., 2010). It has also been shown that pre-extinction DCS administration facilitated fear extinction, although extinguished fear was normally renewed, suggesting that the drug may modestly facilitate extinction learning, but does not destroy the potential for relapse (Woods \& Bouton, 2006). Moreover, the current results contrast with previous research assessing the impact of DCS administered after extinction learning, which indicated enhanced extinction consolidation of different drug-seeking behaviors after intra-BLA or systemic DCS injections (Botreau et al., 2006; Kelley et al., 2007; Nic Dhonnchadha et al., 2009; Paolone et al., 2009; Thanos et al., 2009). Nevertheless, additional reports have also shown other inconsistencies in the capacity of DCS to enhance extinction consolidation. Such inconsistencies may be attributed to previous studies indicating that the effects of DCS may be limited to animals exposed to non-intensive training in CPP (Paolone et al., 2009), or showing low anxiety levels (Tomilenko \& Dubrovina, 2007) or a previously high extinction level (Bouton, Vurbic \& Woods, 2008; Weber, Hart \& Richardson, 2007). The discrepancies between findings suggest that there may be fundamental methodological differences influencing the memory processes activated during retrieval of such different tasks. The fact that DCS shows a complexity of actions, on the grounds of its sensitivity to different procedures, supports the notion that it may not have a pervasive role as an extinction enhancer.

Further factors need to be considered in the interpretation of results regarding vulnerability to extinction, such as memory strength and age, and duration of the reactivation period (i.e. extinction training length). It has been suggested that strong memories are more resistant to extinction (Suzuki et al., 2004). In the present task, odor-reward memory may be considered as a strong memory in that it is based on a survival-based behavior of finding food under food-deprived conditions. Be that as it may, ODT does not involve strong aversive stimuli, such as foot shocks, which may well induce stronger memories. It is also likely that older memories are less susceptible to extinction (Suzuki et al., 2004). However, in the experiment that concerns us here, the memory was relatively recent as the acquisition session took place just one day prior to extinction learning. It has also been suggested that short CS re- 
exposures in the absence of US lead preferentially to reconsolidation rather than to extinction (Lee et al., 2006; Suzuki et al., 2004). Although the extinction session in the present study may be deemed short, the protocol would appear to be sufficient as it included 5-trial sessions lasting approximately 15 min each. However, as the above three features apply to both DCS and VEH groups, with the latter showing marked signs of extinction, intra-BLA DCS may have obstructed the ability to learn behavior extinction, i.e. potentially to form a new CS-no US memory, or may have preserved the original memory.

Experiment 2 involved a brief 90 -sec reactivation period in which the rats were presented with the odor previously associated with a reward. The results indicated that a prereactivation infusion of DCS into the BLA enhanced memory expression in a subsequent 1-day test. The DCS effect seems to be mainly produced on memory expression/reconsolidation as opposed to additional learning or reacquisition during the reconsolidation test session, as the DCS-REACT group showed a better performance in the first trial of the test. Additionally, DCS does not seem to have affected the reinforcer intake during the test as all the rats retrieved and consumed the chocolate cereal in each trial. The experiment also demonstrated that the mere reexposure to the odorous stimulus (VEH-REACT) or the DCS injection (DCS-nonREACT) is not sufficient to notably enhance the odor- reward memory. Our findings, together with other reports, identify memory reactivation as an opportunity to strengthen the memory trace by means of DCS. Thus, intra-BLA DCS has been shown to enhance the reconsolidation of stimulus-cocaine memories as it increased cue-induced relapse in rats with an extensive drug self-administration history (Lee et al., 2009). Moreover, studies carried out in single-trial paradigms have shown that DCS, injected intraperitoneally or into the BLA, potentiates fear memory reconsolidation by increasing the animals' freezing responses during the test (Lee et al., 2006; Yamada et al., 2009). Consistent with our interpretation that a positive modulation of NMDA transmission may help to stabilize the original odor-reward memory trace when reactivated, a previous study showed that intracerebroventricular blockade of NMDARs immediately after reactivation induced amnesia for the stimuli association (Torras-Garcia et al., 2005). Similarly, the administration of the NMDARs antagonist APV within the BLA prior to 
(but not after) a reactivation session, prevented reconsolidation of drug-associated memory, indicating that NMDARs have a temporally limited role in the reconsolidation process (Milton, Lee, Butler, Gardner \& Everitt, 2008).

Although these data suggest that DCS is able to potentiate memory consolidation after reactivation, namely reconsolidation, such an interpretation may be complicated by the fact that the injections were administered pre-reactivation (as in other reconsolidation studies, see Bustos et al., 2010; Lee et al., 2009; Lee et al., 2006; Yamada et al., 2009). Thus, it is possible that the effects found in the reconsolidation test may be due to performance effects on the actual reactivation trial. However, the observation of the animals' behavior during reactivation by the experimenters showed comparable olfactory bouts or motor activity between DCS- and VEHtreated animals. Such observations agree with findings from other studies showing that DCS injected before reactivation did not influence freezing behavior during the reactivation session of fear conditioning (Bustos et al., 2010; Lee et al., 2006). Nevertheless, post-session injections would have better isolated retrieval-induced memory processes, and the possibility that postreactivation DCS affect ODT reconsolidation should be addressed in future studies. In the interpretation of results from experiment 2 , we should also consider the importance of the relation between the glutamatergic transmission in the BLAand reward-related processes (Mead \& Stephens, 2003; Wassum et al., 2012). Thus, our findings may also support the notion that DCS, by increasing glutamate transmission in the BLA, could strengthen the conditioned reinforcing properties of the odor in the conditioned approach response occurring during reactivation.

Although DCS is a commonly studied cognitive enhancer, its mechanisms of action are not completely understood (Davis et al., 2006). Nevertheless, as plasticity in the BLA has been shown as an important factor for reconsolidation and extinction of emotional memories (Duvarci et al., 2006), the effects of DCS have been widely interpreted in terms of modulation of neuroplasticity (Myers \& Davis, 2002). Thus, DCS-induced NMDARs efficacy enhancement, by stimulating high affinity glycine binding, may possibly modify NMDARs-mediated 
intracellular events (Norberg, Krystal \& Tolin, 2008). NMDA agonists and other cognitive enhancers may trigger a signaling cascade resulting in AMPA receptor subunit internalization in the amygdala and enduring alterations in synaptic transmission resulting in changes in the number and morphology of dendritic spines at cortical inputs to amygdalar neurons (S. C. Mao, Lin, \& Gean, 2008; Yang, Chao, Ro, Wo \& Lu, 2007). Particularly noteworthy in such a context is the report that activation of amygdalar protein kinase $\mathrm{A}$, a key component of the synaptic plasticity machinery, enhanced fear memory when the memory was briefly reactivated but not extinguished (Tronson, Wiseman, Olausson, \& Taylor, 2006).

A final issue to be considered is that although the BLA has been extensively related to extinction and reconsolidation of different tasks, other brain regions may also participate in such processes. In this context, several studies have implicated the hippocampus in reconsolidation (Nader \& Hardt, 2009; Tronson \& Taylor, 2007) and the medial prefrontal cortex, which modulates amygdalar activity, in reconsolidation and extinction (Akirav \& Maroun, 2006; Myers \& Davis, 2007; Quirk \& Mueller, 2008; Milton \& Everitt, 2010). As for ODT, a study using c-fos immunocytochemical marking showed that a circuit linking BLA and the prefrontal cortex is involved in the post-acquisition consolidation period of the odor-reward association (Tronel \& Sara, 2002). Also, NMDARs in prelimbic cortex are necessary in the early stage of ODT consolidation as blockade of such receptors immediately after training induced longlasting amnesia (Tronel \& Sara, 2003). This agrees with a recent study demonstrating that bilateral DCS administration in the prelimbic cortex enhanced subsequent ODT memory expression in a relearning test (Villarejo-Rodriguez et al., 2010). Considering this evidence, it is likely that DCS infused in regions anatomically related to BLA and also involved in this task, such as the prefrontal cortex, affect ODT extinction and/or reconsolidation. Hence, additional studies would need to investigate the effects of DCS infusions into different brain regions in order to further explore the neural mechanisms involved in ODT extinction and reconsolidation. Additionally, other circumstances (e.g. re-exposure protocol, dose and timing of injection) would need to be examined to elucidate the potential use of DCS. 


\subsection{Conclusions}

The results presented corroborate the involvement of the NMDARs in an appetitive odor-food reward task (Tronel \& Sara, 2003), and particularly its strychnine-insensitive glycinebinding site (Villarejo-Rodriguez et al., 2010). Specifically, the results show that intra-BLA DCS may exert opposite effects in ODT extinction and reconsolidation, namely, impairment of extinction and enhancement of reconsolidation. Such results suggest that DCS into the BLA may potentiate the persistence or strength of the original odor-reward memory trace (demonstrated by resistance to extinction and facilitation of reacquisition and reconsolidation), which is compatible with the idea that memory retrieval is a dynamic process that either reinforces or alters memory (Suzuki et al., 2004). Therefore, studies of DCS or other agents that may act specifically on extinction or reconsolidation are particularly interesting in order to avoid problematic potential side effects in the therapies used to treat neurologic or psychiatric disorders.

\section{Acknowledgements}

This work was supported by funds from the Ministerio de Ciencia e Innovación (PSI2008-04267; FPI grant to MP-T: BES-2009-015395; Juan de la Cierva grant to PB-V: JCI-2008-2168). The authors thank Mr. Gerald-Patrick Fannon for his support with the Englishlanguage editing and Elena Ilioi for assistance in experiment 2.

\section{References}

Akirav, I., \& Maroun, M. (2006). Ventromedial prefrontal cortex is obligatory for consolidation and reconsolidation of object recognition memory. Cerebral cortex (New York, N.Y.: 1991), 16(12), 1759-65.

Akirav, I., Segev, A., Motanis, H., \& Maroun, M. (2009). D-cycloserine into the BLA reverses the impairing effects of exposure to stress on the extinction of contextual fear, but not conditioned taste aversion. Learning \& memory (Cold Spring Harbor, N.Y.), 16(11), 682-6.

Botreau, F., Paolone, G., \& Stewart, J. (2006). d-cycloserine facilitates extinction of a cocaineinduced conditioned place preference. Behavioural Brain Research, 172(1), 173-178.

Bouton, M. E., Vurbic, D., \& Woods, A. M. (2008). D-cycloserine facilitates context-specific fear extinction learning. Neurobiology of Learning and Memory, 90(3), 504-510.

Bustos, S. G., Giachero, M., Maldonado, H., \& Molina, V. A. (2010). Previous stress attenuates 
the susceptibility to midazolam's disruptive effect on fear memory reconsolidation: Influence of pre-reactivation D-cycloserine administration. Neuropsychopharmacology : Official Publication of the American College of Neuropsychopharmacology, 35(5), 1097-1108.

Carballo-Marquez, A., Vale-Martinez, A., Guillazo-Blanch, G., \& Marti-Nicolovius, M. (2009). Muscarinic receptor blockade in ventral hippocampus and prelimbic cortex impairs memory for socially transmitted food preference. Hippocampus, 19(5), 446-455.

Carballo-Marquez, A., Vale-Martinez, A., Guillazo-Blanch, G., Torras-Garcia, M., Boix-Trelis, N., \& Marti-Nicolovius, M. (2007). Differential effects of muscarinic receptor blockade in prelimbic cortex on acquisition and memory formation of an odor-reward task. Learning \&

Curlik and Shors. (2011). Learning increases the survival of newborn neurons provided that learning is difficult to achieve and successful, 23(9), 2159-2170.

Davis, M. (2002). Role of NMDA receptors and MAP kinase in the amygdala in extinction of fear: Clinical implications for exposure therapy. The European Journal of Neuroscience, 16(3), 395-398.

Davis, M., Ressler, K., Rothbaum, B. O., \& Richardson, R. (2006). Effects of D-cycloserine on extinction: Translation from preclinical to clinical work. Biological Psychiatry, 60(4), 369-375.

de la Fuente, V., Freudenthal, R., \& Romano, A. (2011). Reconsolidation or extinction: Transcription factor switch in the determination of memory course after retrieval. The Journal of Neuroscience : The Official Journal of the Society for Neuroscience, 31(15), $5562-5573$.

Duvarci, S., Mamou, C. B., \& Nader, K. (2006). Extinction is not a sufficient condition to prevent fear memories from undergoing reconsolidation in the basolateral amygdala. The European Journal of Neuroscience, 24(1), 249-260.

Gabriele, A., \& Packard, M. G. (2007). D-cycloserine enhances memory consolidation of hippocampus-dependent latent extinction. Learning \& Memory (Cold Spring Harbor, N.Y.), 14(7), 468-471.

Groblewski, P. A., Lattal, K. M., \& Cunningham, C. L. (2009). Effects of D-cycloserine on extinction and reconditioning of ethanol-seeking behavior in mice. Alcoholism, Clinical and Experimental Research, 33(5), 772-782.

Guastella, A. J., Lovibond, P.F., Dadds, M. R., Mitchell, P., \& Richardson, R. (2007). A randomized controlled trial of the effect of D-cycloserine on extinction and fear conditioning in humans. Behaviour research and therapy, 45(4), 663-72.

Hofmann, S. G. (2007). Enhancing exposure-based therapy from a translational research perspective. Behaviour Research and Therapy, 45(9), 1987-2001.

Kelley, J. B., Anderson, K. L., \& Itzhak, Y. (2007). Long-term memory of cocaine-associated context: Disruption and reinstatement. Neuroreport, 18(8), 777-780.

Kindt, M., Soeter, M., \& Vervliet, B. (2009). Beyond extinction: Erasing human fear responses and preventing the return of fear. Nature Neuroscience, 12(3), 256-258.

Klumpers, F., Denys, D., Kenemans, J. L., Grillon, C., van der Aart, J., \& Baas, J. M. P. (2012). Testing the effects of $\triangle 9$-THC and D-cycloserine on extinction of conditioned fear in humans. Journal of psychopharmacology (Oxford, England), 26(4), 471-8. 
Ledgerwood, L., Richardson, R., \& Cranney, J. (2003). Effects of D-cycloserine on extinction of conditioned freezing. Behavioral Neuroscience, 117(2), 341-349.

Ledgerwood, L., Richardson, R., \& Cranney, J. (2005). D-cycloserine facilitates extinction of learned fear: Effects on reacquisition and generalized extinction. Biological Psychiatry, 57(8), 841-847.

Lee, J. L., Gardner, R. J., Butler, V. J., \& Everitt, B. J. (2009). D-cycloserine potentiates the reconsolidation of cocaine-associated memories. Learning \& Memory (Cold Spring Harbor, N.Y.), 16(1), 82-85.

Lee, J. L., Milton, A. L., \& Everitt, B. J. (2006). Reconsolidation and extinction of conditioned fear: Inhibition and potentiation. The Journal of Neuroscience : The Official Journal of the Society for Neuroscience, 26(39), 10051-10056.

Lu, G. Y., Wu, N., Zhang, Z. L., Ai, J., \& Li, J. (2011). Effects of D-cycloserine on extinction and reinstatement of morphine-induced conditioned place preference. Neuroscience Letters, 503(3), 196-199.

Mao, S. C. C., Hsiao, Y.-H. H., \& Gean, P.-W. W. (2006). Extinction training in conjunction with a partial agonist of the glycine site on the NMDA receptor erases memory trace. The Journal of neuroscience: the official journal of the Society for Neuroscience, 26(35), 8892-8899.

Mao, S. C., Lin, H. C., \& Gean, P. W. (2008). Augmentation of fear extinction by D-cycloserine is blocked by proteasome inhibitors. Neuropsychopharmacology: Official Publication of the American College of Neuropsychopharmacology, 33(13), 3085-3095.

Mead, A. N., \& Stephens, D. N. (2003). Selective disruption of stimulus-reward learning in glutamate receptor gria1 knock-out mice. The Journal of neuroscience: the official journal of the Society for Neuroscience, 23(3), 1041-8.

Mickley, G. A., Remus, J. L., Ramos, L., Wilson, G. N., Biesan, O. R., \& Ketchesin, K. D. (2011). Acute, but not chronic, exposure to d-cycloserine facilitates extinction and modulates spontaneous recovery of a conditioned taste aversion. Physiology \& behavior, 105(2), 417-427.

Milton, A. L., \& Everitt, B. J. (2010). The psychological and neurochemical mechanisms of drug memory reconsolidation: Implications for the treatment of addiction. The European Journal of Neuroscience, 31(12), 2308-2319.

Milton, A. L., Lee, J. L., Butler, V. J., Gardner, R., \& Everitt, B. J. (2008). Intra-amygdala and systemic antagonism of NMDA receptors prevents the reconsolidation of drug-associated memory and impairs subsequently both novel and previously acquired drug-seeking behaviors. The Journal of Neuroscience : The Official Journal of the Society for Neuroscience, 28(33), 8230-8237.

Myers, K. M., \& Davis, M. (2002). Behavioral and neural analysis of extinction. Neuron, 36(4), 567-584.

Myers, K. M., \& Davis, M. (2007). Mechanisms of fear extinction. Molecular Psychiatry, 12(2), $120-150$.

Nader, K., \& Hardt, O. (2009). A single standard for memory: The case for reconsolidation. Nature Reviews.Neuroscience, 10(3), 224-234.

Nader, K., Schafe, G. E., \& Le Doux, J. E. (2000). Fear memories require protein synthesis in the amygdala for reconsolidation after retrieval. Nature, 406(6797), 722-726. 
Nic Dhonnchadha, B. A., Szalay, J. J., Achat-Mendes, C., Platt, D. M., Otto, M. W., Spealman, R. D., \& Kantak, K. M. (2010). D-cycloserine deters reacquisition of cocaine selfadministration by augmenting extinction learning. Neuropsychopharmacology : Official Publication of the American College of Neuropsychopharmacology, 35(2), 357-367.

Norberg, M. M., Krystal, J. H., \& Tolin, D. F. (2008). A meta-analysis of D-cycloserine and the facilitation of fear extinction and exposure therapy. Biological Psychiatry, 63(12), $1118-1126$.

Paolone, G., Botreau, F., \& Stewart, J. (2009). The facilitative effects of D-cycloserine on extinction of a cocaine-induced conditioned place preference can be long lasting and resistant to reinstatement. Psychopharmacology, 202(1-3), 403-409.

Paxinos, G., \& Watson, C. (1997). The rat brain in stereotaxic coordinates. San Diego: Academic Press,

Pedreira, M. E., \& Maldonado, H. (2003). Protein Synthesis Subserves Reconsolidation or Extinction Depending on Reminder Duration. Neuron, 38(6), 863-869.

Port, R. L., \& Seybold, K. S. (1998). Manipulation of NMDA-receptor activity alters extinction of an instrumental response in rats. Physiology \& Behavior, 64(3), 391-393.

Price, K. L., Baker, N. L., McRae-Clark, A. L., Saladin, M. E., Desantis, S. M., Santa Ana, E. J., \& Brady, K. T. (2012). A randomized, placebo-controlled laboratory study of the effects of D: -cycloserine on craving in cocaine-dependent individuals. Psychopharmacology.

Quirk, G. J., \& Mueller, D. (2008). Neural mechanisms of extinction learning and retrieval. Neuropsychopharmacology: Official Publication of the American College of Neuropsychopharmacology, 33(1), 56-72.

Quiroz-Padilla, M. F., Guillazo-Blanch, G., Vale-Martinez, A., \& Marti-Nicolovius, M. (2006). Excitotoxic lesions of the parafascicular nucleus produce deficits in a socially transmitted food preference. Neurobiology of Learning and Memory, 86(3), 256-263.

Quiroz-Padilla, M. F., Guillazo-Blanch, G., Vale-Martinez, A., Torras-Garcia, M., \& MartiNicolovius, M. (2007). Effects of parafascicular excitotoxic lesions on two-way active avoidance and odor-discrimination. Neurobiology of Learning and Memory, 88(2), 198-207.

Rehberg, K., Bergado-Acosta, J. R., Koch, J. C., \& Stork, O. (2010). Disruption of fear memory consolidation and reconsolidation by actin filament arrest in the basolateral amygdala. Neurobiology of Learning and Memory, 94(2), 117-126.

Rodgers, R. J., Harvest, H., Hassall, C., \& Kaddour, L. A. (2011). D-cycloserine enhances memory consolidation in the plus-maze retest paradigm. Behavioral Neuroscience, 125(1), 106-116.

Storch, E. a, Murphy, T. K., Goodman, W. K., Geffken, G. R., Lewin, A. B., Henin, A., Micco, J. a, et al. (2010). A preliminary study of D-cycloserine augmentation of cognitivebehavioral therapy in pediatric obsessive-compulsive disorder. Biological psychiatry, 68(11), 1073-6.

Suzuki, A., Josselyn, S. A., Frankland, P. W., Masushige, S., Silva, A. J., \& Kida, S. (2004). Memory reconsolidation and extinction have distinct temporal and biochemical signatures. The Journal of Neuroscience : The Official Journal of the Society for Neuroscience, 24(20), 4787-4795.

Thanos, P. K., Bermeo, C., Wang, G. J., \& Volkow, N. D. (2009). D-cycloserine accelerates the 
extinction of cocaine-induced conditioned place preference in $\mathrm{C} 57 \mathrm{bL} / \mathrm{c}$ mice. Behavioural Brain Research, 199(2), 345-349.

Thanos, P. K., Bermeo, C., Wang, G. J., \& Volkow, N. D. (2011). D-cycloserine facilitates extinction of cocaine self-administration in rats. Synapse (New York, N.Y.)

Tomilenko, R. A., \& Dubrovina, N. I. (2007). Effects of activation and blockade of NMDA receptors on the extinction of a conditioned passive avoidance response in mice with different levels of anxiety. Neuroscience and Behavioral Physiology, 37(5), 509-515.

Torras-Garcia, M., Lelong, J., Tronel, S., \& Sara, S. J. (2005). Reconsolidation after remembering an odor-reward association requires NMDA receptors. Learning \& Memory (Cold Spring Harbor, N.Y.), 12(1), 18-22.

Torregrossa, M. M., Sanchez, H., \& Taylor, J. R. (2010). D-cycloserine reduces the context specificity of pavlovian extinction of cocaine cues through actions in the nucleus accumbens. The Journal of neuroscience: the official journal of the Society for Neuroscience, 30(31), 10526-33.

Toth, I., Dietz, M., Peterlik, D., Huber, S. E., Fendt, M., Neumann, I. D., Flor, P. J., et al. (2012). Pharmacological interference with metabotropic glutamate receptor subtype 7 but not subtype 5 differentially affects within- and between-session extinction of Pavlovian conditioned fear. Neuropharmacology, 62(4), 1619-26.

Tronel, S., \& Sara, S. J. (2002). Mapping of olfactory memory circuits: Region-specific c-fos activation after odor-reward associative learning or after its retrieval. Learning \& Memory (Cold Spring Harbor, N.Y.), 9(3), 105-111.

Tronel, S., \& Sara, S. J. (2003). Blockade of NMDA receptors in prelimbic cortex induces an enduring amnesia for odor-reward associative learning. The Journal of Neuroscience : The Official Journal of the Society for Neuroscience, 23(13), 5472-5476.

Tronson, N. C., Wiseman, S. L., Olausson, P., \& Taylor, J. R. (2006). Bidirectional behavioral plasticity of memory reconsolidation depends on amygdalar protein kinase A. Nature neuroscience, 9(2), 167-169.

Tronson, N. C., \& Taylor, J. R. (2007). Molecular mechanisms of memory reconsolidation. Nature Reviews.Neuroscience, 8(4), 262-275.

Villarejo-Rodriguez, I., Vale-Martinez, A., Guillazo-Blanch, G., \& Marti-Nicolovius, M. (2010). d-cycloserine in prelimbic cortex enhances relearning of an odor-reward associative task. Behavioural Brain Research.

Vurbic, D., Gold, B., \& Bouton, M. E. (2011). Effects of D-cycloserine on the extinction of appetitive operant learning. Behavioral Neuroscience, 125(4), 551-559.

Walker, D. L., Ressler, K. J., Lu, K. T., \& Davis, M. (2002). Facilitation of conditioned fear extinction by systemic administration or intra-amygdala infusions of D-cycloserine as assessed with fear-potentiated startle in rats. The Journal of Neuroscience : The Official Journal of the Society for Neuroscience, 22(6), 2343-2351.

Wassum, K. M., Tolosa, V. M., Tseng, T. C., Balleine, B. W., Monbouquette, H. G., \& Maidment, N. T. (2012). Transient Extracellular Glutamate Events in the Basolateral Amygdala Track Reward-Seeking Actions. The Journal of neuroscience: the official journal of the Society for Neuroscience, 32(8), 2734-2746.

Watson, B. J., Wilson, S., Griffin, L., Kalk, N. J., Taylor, L. G., Munafò, M. R., LingfordHughes, a. R., et al. (2011). A pilot study of the effectiveness of d-cycloserine during cue- 
exposure therapy in abstinent alcohol-dependent subjects. Psychopharmacology, 216(1), 121-129.

Weber, M., Hart, J., \& Richardson, R. (2007). Effects of D-cycloserine on extinction of learned fear to an olfactory cue. Neurobiology of Learning and Memory, 87(4), 476-482.

Woods, A. M., \& Bouton, M. E. (2006). D-cycloserine facilitates extinction but does not eliminate renewal of the conditioned emotional response. Behavioral neuroscience, $120(5), 1159-62$.

Wrenn, C. C., Harris, A. P., Saavedra, M. C., \& Crawley, J. N. (2003). Social transmission of food preference in mice: Methodology and application to galanin-overexpressing transgenic mice. Behavioral Neuroscience, 117(1), 21-31.

Yamada, D., Zushida, K., Wada, K., \& Sekiguchi, M. (2009). Pharmacological discrimination of extinction and reconsolidation of contextual fear memory by a potentiator of AMPA receptors. Neuropsychopharmacology: Official Publication of the American College of Neuropsychopharmacology, 34(12), 2574-2584.

Yamamoto, S., Morinobu, S., Fuchikami, M., Kurata, A., Kozuru, T., \& Yamawaki, S. (2008). Effects of single prolonged stress and D-cycloserine on contextual fear extinction and hippocampal NMDA receptor expression in a rat model of PTSD.

Neuropsychopharmacology: Official Publication of the American College of Neuropsychopharmacology, 33(9), 2108-2116.

Yang, Y. L., Chao, P. K., Ro, L. S., Wo, Y. Y., \& Lu, K. T. (2007). Glutamate NMDA receptors within the amygdala participate in the modulatory effect of glucocorticoids on extinction of conditioned fear in rats. Neuropsychopharmacology : Official Publication of the American College of Neuropsychopharmacology, 32(5), 1042-1051. 


\section{Figure Captions}

Figure 1. (A) Photomicrograph (2× magnification) of acetylcholinesterase staining at the level of BLA (AP, $3.14 \mathrm{~mm}$ posterior to bregma) showing the cannula track and the microinjector tip of a representative subject [BLA, basolateral amygdala, $\mathrm{CPu}$, caudate-putamen; $\mathrm{La}$, lateral amygdala; Me, medial amygdala]. (B, C) Location of injectors within the BLA. Schematic representation of the brain at four rostro-caudal levels $(-2.30,-2.56,-2.80$ and $\cdot 3.14 \mathrm{~mm}$ from bregma). PBS-infused rats are represented by empty circles and DCS-infused rats by filled circles, for experiment 1(B) and experiment 2 (C).

Figure 2. Effects of pre-extinction intra-BLA DCS on the odor-reward task (experiment 1). $(A)$ The behavioral procedure used for experiment $1,(B)$ Latency to make the original correct response $( \pm \mathrm{SEM})$ in each session $(\mathrm{C})$ Trial-by-trial analysis of the latency to make the original correct response. (D) Number of total errors prior to making the original correct response $( \pm$ SEM) in each session (E) Trial-by-trial analysis of the number of total errors. DCS significantly decreased both measures in the extinction learning and extinction retention and improved the reacquisition of the task $(* \mathrm{p}<0.05, * * \mathrm{p}<0.05, * * * \mathrm{p}<0.001)$, suggesting a weakened extinction learning.

Figure 3. Survival analysis of extinction learning and retention (experiment 1). The percentage of subjects reaching the extinction criterion (latency to make the original correct response $\geq$ total mean latency of the first acquisition trial, 44 secs, for two consecutive trials) is shown in the $\mathrm{Y}$-axis ( 1 indicates that $100 \%$ of subjects acquired the criterion) for each of the 5 trials (Xaxis) in each session. A significantly higher percentage of VEH subjects achieved the criterion during extinction. .

Figure 4. Effects of pre-reactivation intra-BLA DCS on the odor-reward task (experiment 2).

(A) The behavioral procedure used for experiment 2, $(B)$ Latency to make the correct response in each session $( \pm$ SEM) and $(\mathrm{C})$ Trial-by-trial analysis of the latency to make the correct response in the test session. (D) Number of total errors prior to making the correct response $( \pm$ SEM). (E) Trial-by-trial analysis of the number of total errors in the test session. DCS in the 
reactivated rats significantly decreased both measures throughout the test session, in contrast to the remaining groups $(* \mathrm{p}<0.05, * * \mathrm{p}<0.05, * * * p<0.001)$, which indicated an enhanced reconsolidation. 